Military Technical College

Kobry Elkobbah,

Cairo, Egypt

May 25-27,2010

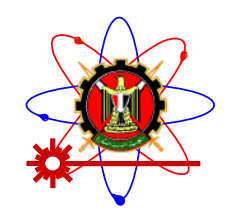

$5^{\text {th }}$ International Conference on Mathematics and Engineering Physics (ICMEP-5)

PH-25

\title{
Studying the Thermal Effects on Operation and Wavelength Stability of High Power Fiber Coupled Diode Lasers operating at $808 \mathrm{~nm}$ and $975 \mathrm{~nm}$
}

\author{
Ashraf F. El-Sherif ${ }^{1}$, M. Kh. El-Tahlawy ${ }^{2}$, and M. F. Hassan
}

\begin{abstract}
A complete characterization and comparison of thermal effect between two high power fiber coupled diode laser modules operating at wavelengths of $808 \mathrm{~nm}$ and $975 \mathrm{~nm}$. For the diode laser to operate at certain wavelength, the internal construction and the used material inside the diode must be different. Both wavelengths have a wide use in the laser design such as pumping another active medium to be lase at a new wavelength, $808 \mathrm{~nm}$ used to excite solid state lasers such as Nd:YVO and $975 \mathrm{~nm}$ used to excite fiber laser such as $\mathrm{Yb}$ fiber laser and $\mathrm{Er}-\mathrm{Yb}$ fiber lasers, and also in applications in several fields such as military, industrial and biomedical engineering fields. For the $808 \mathrm{~nm}$ diode laser the output wavelength varied with changing the temperature while the $975 \mathrm{~nm}$ has a very large stability against the change of the operating temperature. We obtained an optical power up to 6 Watt $(\mathrm{cw})$. The electrical characterization of the diode laser modules was examined by measuring the dependence of the laser driving current on the operating voltage. The optical characterization was investigated at different temperatures. These included the laser output power versus the driving current, the output optical power versus electrical input power, the laser output spectrum, the laser beam profile and the dependence of the output power on the temperature. The measured diode laser of $975 \mathrm{~nm}$ parameters at $25^{\circ} \mathrm{C}$ were $(0.3 \mathrm{~A})$ threshold current, $(35.84 \%)$ overall slope efficiency and $(975.34 \mathrm{~nm})$ central wavelength with line width of $(1.3 \mathrm{~nm})$ at FWHM. The variation of the output power, spectrum with temperature are presented. The measured diode laser of $808 \mathrm{~nm}$ parameters at $25^{\circ} \mathrm{C}$ were (1.3A) threshold current, (42\%) overall slope efficiency and $(807.96 \mathrm{~nm})$ central wavelength with line width of $(3.59 \mathrm{~nm})$ at FWHM. The variation of the above mentioned parameters with temperature are presented
\end{abstract}

\footnotetext{
1,2 Engineering Physics Department, Military Technical College, Cairo, Egypt.

${ }^{3}$ The National Institute of Laser Enhanced Sciences, Cairo University
} 


\section{Introduction:}

Laser diodes (= diode lasers) are electrically pumped semiconductor lasers in which the gain is generated by an electrical current flowing through a $\mathrm{p}-\mathrm{n}$ junction or (more frequently) a $\mathrm{p}-\mathrm{i}-\mathrm{n}$ structure. In such a heterostructure, electrons and holes can recombine, releasing the energy portions as photons. This process can be spontaneous, but can also be stimulated by incident photons, in effect leading to optical amplification, and with optical feedback in a laser resonator to laser oscillation. The article on semiconductor lasers describes more in detail how the laser amplification process in a semiconductor works.

Laser diodes are normally built as edge-emitting lasers, where the laser resonator is formed by coated or uncoated end facets (cleaved edges) of the semiconductor wafer. They are often based on a double heterostructure, which restricts the generated carriers to a narrow region and at the same time serves as a waveguide for the optical field (double confinement). The current flow is restricted to the same region, sometimes using isolating barriers. Such arrangements lead to a relatively low threshold pump power and high efficiency. The active region is usually quite thin - often so thin that it acts as a quantum well. In some cases, quantum dots are used [1]. Laser diodes may emit a beam into free space, but many LDs are also available in fiber-coupled form as in our devices used in the experimental work in this paper. The latter makes it particularly convenient to use them, e.g., as pump sources for fiber lasers and fiber amplifiers.

The emission wavelength of a laser diode is essentially determined by the bandgap of the laser-active semiconductor material: the photon energy is close to the bandgap energy. In quantum well lasers, there is also some influence of the quantum well thickness. A variety of semiconductor materials makes it possible to cover wide spectral regions.

In particular, there are many ternary and quaternary semiconductor compounds, where the bandgap energy can be adjusted in a wide range simply by varying the composition details. For example, an increased aluminum content (increased $\mathrm{x}$ ) in AlxGa1-xAs causes an increase in the bandgap energy and thus a shorter emission wavelength. The used materials in the diode laser to generate the wavelengths of $808 \mathrm{~nm}$ and $975 \mathrm{~nm}$ are AlGaAs (its emission band from $720-850 \mathrm{~nm}$ ) and InGaAs (its emission band from $900-1100 \mathrm{~nm}$ ), respectively. Both wavelengths are in the near-infrared spectral region [1].

The efficiency is usually limited by factors such as the electrical resistance, carrier leakage, scattering, absorption (particularly in doped regions), and spontaneous emission. Particularly high efficiencies are achieved with laser diodes emitting e.g. around 940-980 nm (as used e.g. for pumping ytterbium-doped high-power fiber devices), whereas 808-nm diodes are somewhat less efficient [1].

Most higher-power LDs, however, exhibit a relatively poor beam quality, combined with other non-favorable properties, such as a large beam divergence, high asymmetry of beam $\underline{\text { radius }}$ and beam quality between two perpendicular directions, and astigmatism. It is not always trivial to find the best design for beam shaping optics, being compact, easy to manufacture and align, preserving the beam quality and avoiding interference fringes, removing astigmatism, having low losses, etc.

Typical parts of such diode laser beam shaping optics are collimating lenses (spherical or cylindrical), apertures, and anamorphic prisms. The developing of $\mathrm{TEM}^{00}$ laser mode with high efficiency, high output power, good spatial beam profile and good stability is highly desired. This will make it suitable for using in material processing and other scientific applications. One of these applications is pumping other laser crystals like Nd:YAG and $\mathrm{Nd}: \mathrm{YVO}^{4}$, (Diode-Pumped-Solid-State Laser "DPSSL") with $808 \mathrm{~nm}$ diode laser modules [24], and pumping rare earth doped fibers like $\mathrm{Yb}^{3+}$ and $\mathrm{Er}^{3+}$-doped fiber lasers and $\mathrm{Er}: \mathrm{Yb}$ codoped fiber laser, (Diode-Pumped-Fiber-Laser "DPFL") with $975 \mathrm{~nm}$ diode laser modules [5-11]. 
The new technique such as High Power Diode Laser modules for pumping solid state lasers and fiber lasers is a reliable technique in developing TEM $^{00}$ laser output [12-14]. High power laser diode arrays have found wide applications in manufacturing, defense, communications, and pumping of solid state lasers and fiber lasers.

High power diode laser modules typically have a broad spectral width of about 3 to $6 \mathrm{~nm}$ [15]. In addition, the center wavelength shifts by changing the temperature or the driving current, which is obstructive for pumping applications with small bandwidth. Wavelength of high power laser modules is an important means for more efficient pumping of solid state lasers with narrow bandwidth. However, for efficient and reliable wavelength stabilization the parameters of Volume Holographic Grating (VHG) and the diode laser bars have to be adopted carefully. Important parameters are the reflectivity of the VHG, the reflectivity of the diode laser bar and the angular and spectral emission characteristics of diode laser bar. In addition, the lateral structure of diode laser bar and microoptical elements for beam shaping have to be considered [15].

The diode laser bar is mounted onto a heat sink to remove the waste heat generated by the device under operation. The cooling for the high power diode laser bars can be passive or active. A typically passively cooled diode is sold on a CS mount, a standard package that is compatible with a thermo-electric cooler (TEC) based mounting fixture.

In this paper, we have investigated full electrical and optical characterizations of the main two important high power fiber coupled diode laser modules operating at $808 \mathrm{~nm}$ and $975 \mathrm{~nm}$, respectively. These characterizations can be divided into five categories and we will focus on the highlighting ones:

- Electrical: Measurement of light output, voltage drop, and operating current.

- Spatial: Output light intensity profile in the far and near the field and pointing angle of radiation pattern.

- Spectral: Spectral data acquired to calculate spectral width, center wavelength and to observe mode structure.

- Optical: Measurement of astigmatism and other wave front errors.

- Dynamic: Measurement of noise, intermodulation, distortion, rise time, fall time, chirping and so on.

- Thermal: The variable of the above characteristics with temperature.

\section{Basic Concepts of Wavelength Stabilization:}

\subsection{Approaches of Wavelength Stabilization}

Different methods have been investigated in the past for improving the spectral brightness of diode laser bars. These approaches can be divided into internal and external solutions. For internal solutions the wavelength stabilizing structure is integrated into diode laser bar itself, whereas for external solutions separate bulk elements with integrated Bragg grating are used for wavelength stabilization.

An example for a diode laser bar with internal wavelength stabilization is a distributed feedback diode laser (DFB) where the grating for selective spectral feedback is integrated in the structure of the active region of the laser bar itself. With such a device the wavelength shift with temperature is reduced down to about $0.08 \mathrm{~nm} /{ }^{\circ} \mathrm{C}$ and in addition the spectral bandwidth is reduced to less than $1 \mathrm{~nm}$ [16-18]. It is evident that the fabrication process of such a DFB-diode laser bar is more complex leading to an increase in costs. Another drawback is the reduced efficiency of a DFB diode laser, when compared to standard broad area diode laser bar.

Another drawback of high power diode laser systems with broad area diode laser bars is their relatively poor beam quality and brightness B as defined in equation (1): [19] 


$$
B=\frac{P}{\pi^{2} \cdot B P P^{2}} ; \quad B P P_{\text {total }}=B P P_{\text {slow }}^{2}+B P P_{\text {fast }}^{2}
$$

The brightness of a diode laser beam is defined by the laser power $\mathrm{P}$ and the beam parameter product (BPP - equation (2)) in slaw- and fast-axis-direction [19]

$B P P=\omega_{0} \cdot \theta$

(half beam waist diameter $\frac{d_{0}}{2}$ times half far field divergence angle $\theta$ )

(2)

The output beam of a common broad area diode laser bar is characterized by a highly asymmetric profile with regard to beam dimension and divergence angle. Whereas the beam quality in the fast-direction is about $1 \mathrm{~mm} . \mathrm{mrad}$ and thus nearly diffraction limited, the beam quality of a standard $10 \mathrm{~mm}$ broad area diode laser bar in the slow-direction is in the region 400-500 mm.mrad, which is far beyond the diffraction limit [19].

In recent years the brightness of diode laser bars has been significantly improved mainly by increasing the output power per emitter and by reducing the slow-axis divergence. The development led to the design of new types of diode laser bars with reduced number of emitters and increased pitch between the emitters. These minibars have advantages compared to the traditional $10 \mathrm{~mm}$ broad diode laser bars [20].

In contrast to thin internal approach wavelength stabilization by external components has also been investigated. One example for an external wavelength stabilizing element is a thick volume grating based on a photo thermo refractive (PTR) inorganic glass. Recording of highly efficient Bragg gratings in such photosensitive glass is achieved by periodic variation of the refractive index by UV exposure. Such volume diffractive gratings are commercially available from different vendors with slightly different nomenclatures, like volume Bragg gratings (VBG) [21], volume holographic grating (VHG) [22], or volume Bragg grating laser (VOBLA) [23], whereas the latter also includes the diode laser source.

Because of the angular sensitivity of VHG it is advantages to reduce the divergence of the diode laser bar especially in the fast-axis direction by collimating the beam with a fast-axis collimating lens (FAC). This will significantly increase the optical feedback by VHG. Collimation of the beam in the slow-axis with a SAC is not mandatory. The VHG is positioned directly behind the FAC. The table in Figure (1) shows typical alignment tolerances that are required for efficient wavelength stabilization.

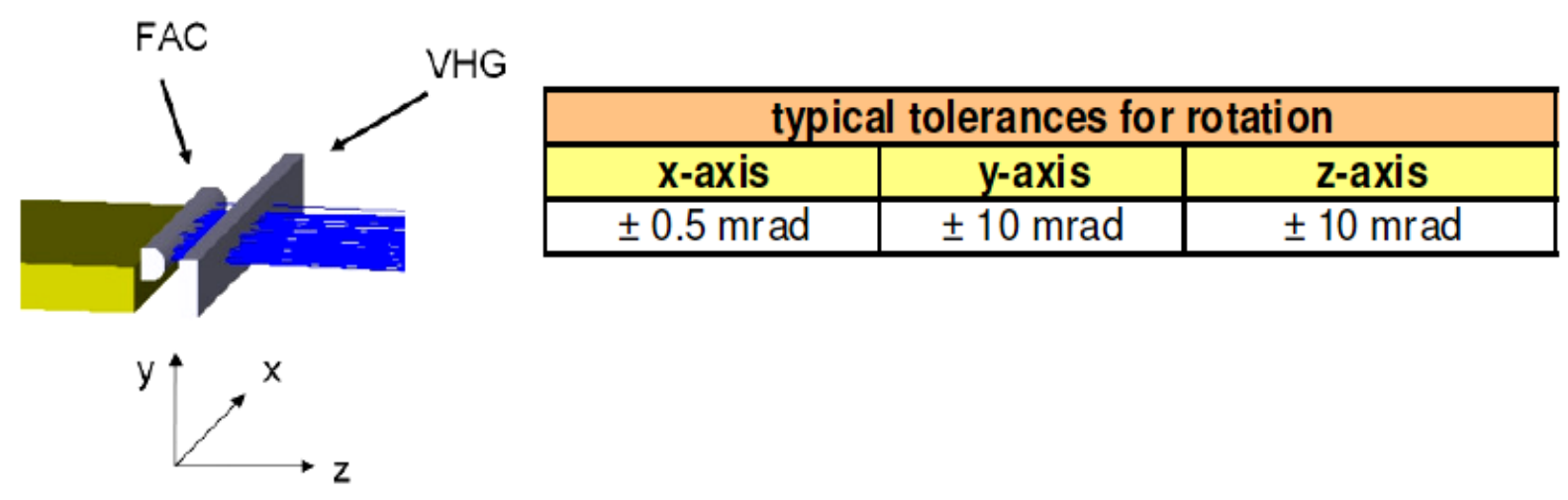

Fig. 1 Typical setup for a wavelength stabilized diode laser bar with VHG positioned directly behind the FAC. The table shows typical alignment tolerance with respect to the shown setup 


\subsection{Influence of diode parameters on the performance of external wavelength stabilization:}

For efficient and stable operation of wavelength stabilization all relevant parameters have to be controlled carefully. The parameters of the diode laser bar include the reflectivity of the AR-coating of the output facet, the emitter structure, the cavity length, the smile, the angular emission characteristics and mounting technology, which has an influence on the wavelength drift with driving current and temperature [15].

The properties of VHG are optimized by adopting the reflective index modulation, the spatial frequency and the thickness. These three independent parameters define the Bragg angle, the diffraction efficiency and the spectral and angular selectivity of the grating. In principle, for each configuration these VHG parameters have to be optimized separately. However, based on the experience a value for the VHG reflectivity of about $20 \%$ is a good starting point for most common diode laser bars [15].

As a matter of course, inserting a VHG for wavelength stabilization will reduce the output power for a given current when compared to the diode bar without stabilization. A VHG with a higher reflectivity will increase the locking range of wavelength in the expense of the higher power loss. This means that optimization of wavelength stabilization will always be a tradeoff between locking range and power loss. Furthermore it is important to notice that the optimum reflectivity also depends on the demands of the application. For some application the VHG has to be optimized for large locking range, whereas for other applications low losses for fixed operating conditions could be requested [15].

The most common setup for wavelength stabilization with an external VHG is a separate bulk VHG positioned directly behind a fast-axis collimation lens (FAC). One important disadvantages of this setup is its sensitivity for smile. As a consequence of smile some emitters are not exactly on the optical axis leading to a deflection angle after collimation and finally to a displacement of the reflected light with respect to the initial position of the emitter. The emitter that is not exactly located on the optical axis will receive less optical feedback as shown in the right diagram of Figure (2).

One means to overcome the sensitivity for smile is the integration of grating structure into the FAC itself [24]. Such an element is more insensitive to smile or misalignment. Due to the large angular divergence of the uncollimated beam and the small angular selectivity of the grating only a small part of the beam is reflected back into the diode bar laser cavity. In the case of misalignment or smile another part of the beam will be reflected to provide feedback. In contrast, for an ideal two components setup with good collimation and no smile nearly all light reflected from the VHG is coupled back into the diode laser cavity. On the other hand this implies that for efficient wavelength locking a significant increase of the reflectivity of VHG-FAC to about $70 \%$ [24].

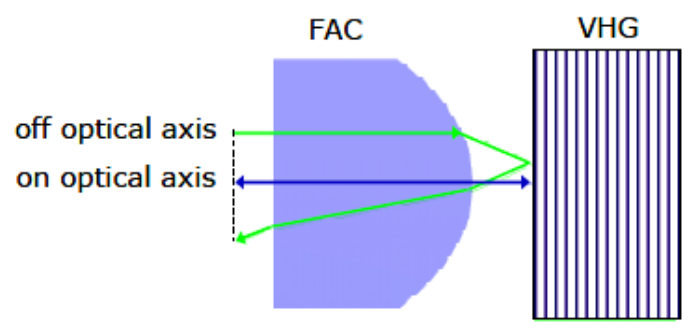

Diode bar with smile

0900000000000000000000000
Reflected Intensity

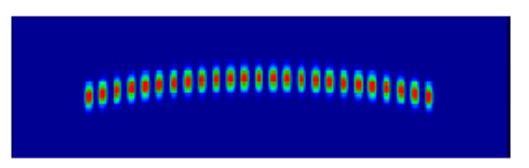

Optical feedback by VHG

IInooponIIIIIIIIII0000001| optical axis 
Fig. 2 Influence of smile on the optical feedback of diode laser bar with volume holographic grating (VHG) for wavelength stabilization.

\section{Electrical Characterization of the Fiber Coupled Diode Module: \\ 3.1 Experimental Setup:}

The experimental setup shown in Figure (3) consists of; laser diode driver with a temperature controller (fully controlled by computer via wireless commands), power supply (output $24 \mathrm{~V}$, 10.5A), high power diode laser module (fiber coupled), optical power meter, spectrometer with integrated sphere, thermal camera and laser optical detectors.

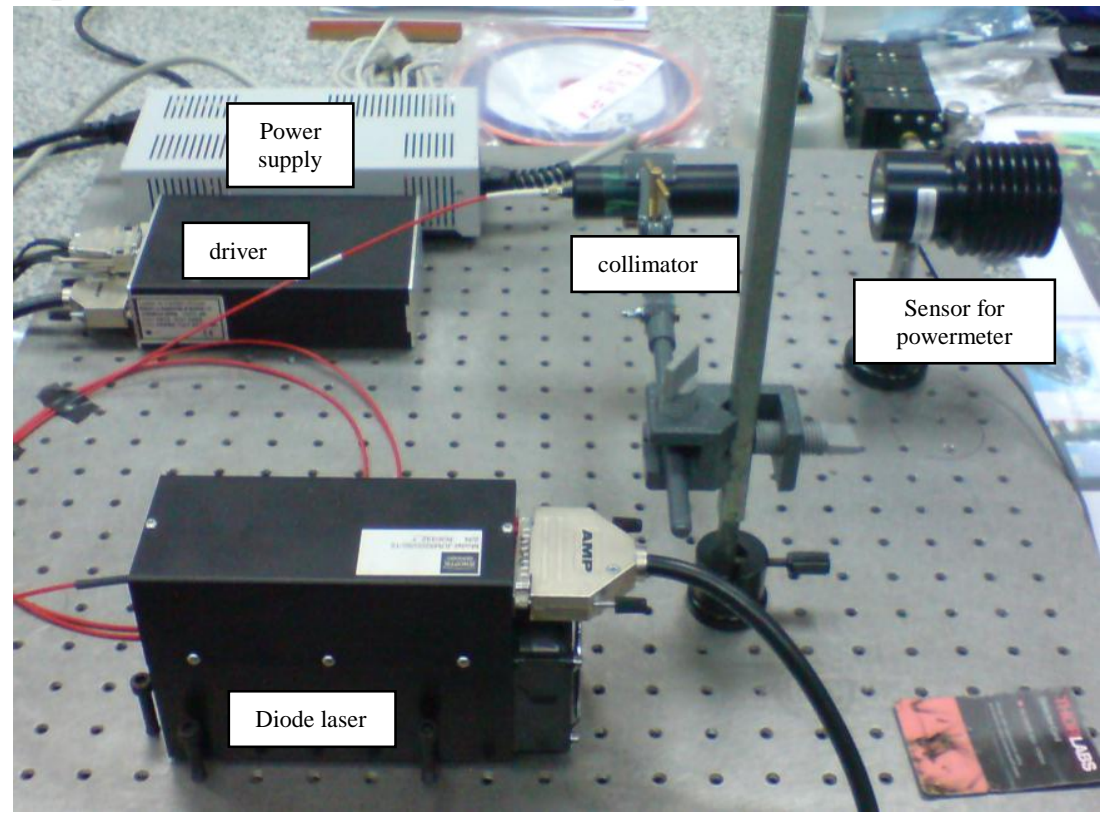

Fig. 3 Photograph of the experimental setup

A laser diode driver (UM DiTec 60/8000 TEC OEM) with an integrated highly precise temperature controller (TEC) used for driving diode lasers in $\mathrm{cw}$ and pulsed mode as it has an On-Board Oscillator which can be freely configure for pulsation the laser. The driver is completely controllable by RS232 serial interface; where the current, voltage and temperature can freely set, as it will be used to measure the electrical and optical characterization of the high power Diode laser module.

Our high power Diode laser module is a fiber coupled @ (808 and $975 \mathrm{~nm}) \pm 2 \mathrm{~nm}$. The highest brightness is achieved by transforming the asymmetric radiation from the laser diode into a symmetrical beam, using micro optics and finally this beam can be coupled into $125 \mu \mathrm{m}$ fiber with core diameter of $50 \mu \mathrm{m}$.

While the optical power meter system (PM213) provided by THORLABS with two different detectors; (S120B) silicon sensor has spectral band ranging from 400:1100nm measuring an optical power ranging form $50 \mathrm{nW}-50 \mathrm{~mW}$ and the other (S213A) Thermal sensor has spectral band ranging from 250:1064nm measuring up to $30 \mathrm{~W}$. Using the silicon sensor for low power measurement since its response is a wavelength dependent.

\subsection{Experimental Results:}

By adjusting the laser diode driver to different voltages and obtaining the driving input current to the diode module (measured and viewed through the computer's screen) at different operating temperature; the measured (I-V) diode characteristic curve is shown in Figure (3). 


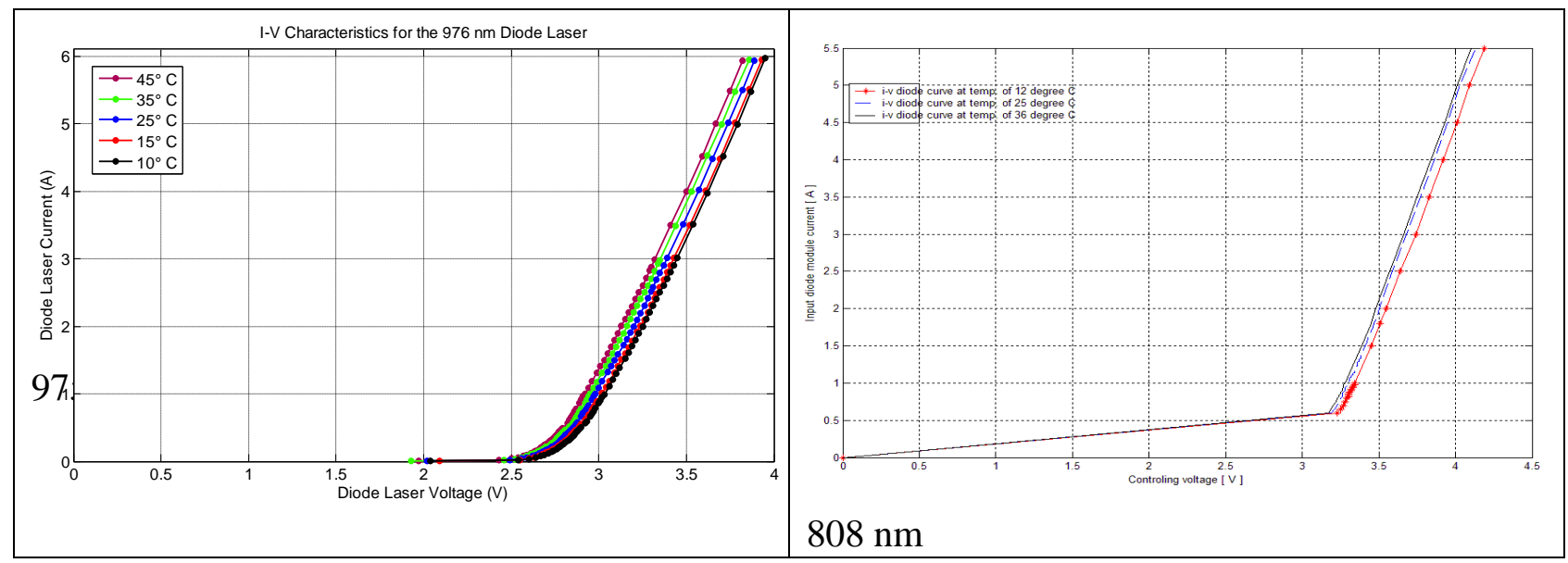

Fig. 4 The (I-V) curve measured at different temperatures

From Figure (4) one can notice that; the maximum consumption electrical power was found at temperature of $10^{\circ} \mathrm{C}$ provided the lowest electrical to optical slope efficiency in this case.

\subsection{Optical Characterization of the Fiber Coupled Diode Laser Module:}

The experimental setup was shown in previous section.

i) Principles and concepts of the measurements:

The light emitted by one facet of the semiconductor (Diode) laser was measured as a function of the driver input current (I); and the measuring curve is referred to as the Light-Current (L-I) curve which is strongly temperature dependent. The form of the (L-I) curve is typical the same as in any lasers; the turning point at which the laser output abruptly start to increase corresponds to the threshold lasing point. The threshold current $\left(\mathrm{I}_{\mathrm{th}}\right)$ or equivalently threshold current density $\left(\mathrm{J}_{\mathrm{th}}\right)$ is an important device parameter and its minimization is desirable. It is well known that when the input current $\mathrm{I}<\mathrm{I}_{\mathrm{th}}$; light output mainly consists of spontaneous emission.

ii) Optical characterization measurements results:

The output laser power at a constant operating temperature $\left(25^{\circ} \mathrm{C}\right)$ is measured using the optical power meter and the laser detectors; corresponding to the change in the driving input current to the Diode laser module. As the optical characteristics (L-I) curve can be investigated. Diode output laser power (Watt) Vs. the diode input driving current (A); measured at different temperatures.

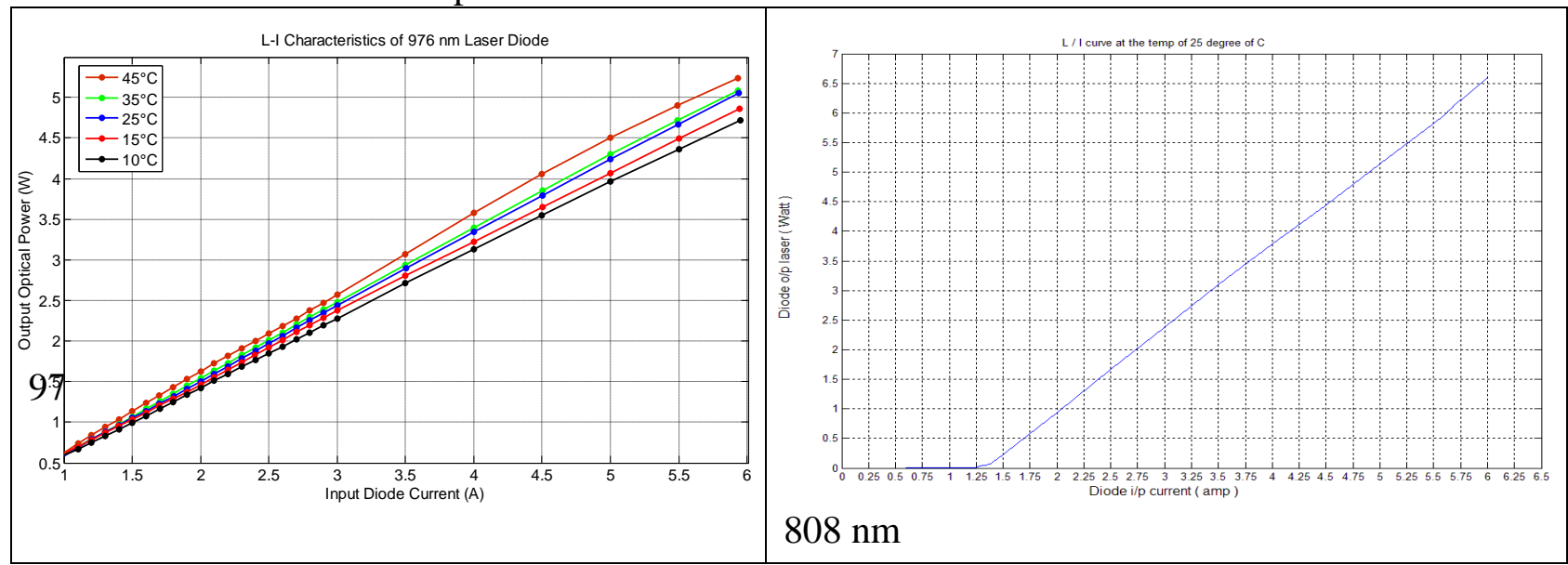

Fig. 5 The Optical characteristics (L-I) curve of the high power diode module at operating temperature of different values 
Output measured threshold laser was $528 \mu \mathrm{W}$ at input driving current of 0.3A (using S120B photo detector for measuring such low output power) for the $975 \mathrm{~nm}$ and $45.42 \mathrm{~mW}$ at $1.3 \mathrm{~A}$ input current for the $808 \mathrm{~nm}$ diode lasers.

The (L-I) characteristic curve is measured at different temperatures showing the temperature effect on the threshold lasing point and the slope efficiency shown in the next section. Figure (5) shows the clearly linear relation between the output laser power and the input driving current behind threshold point, as the laser is shown to operate continuously without any indication of heating or failure.

The conversion efficiency of the pigtail fiber was discussed by measuring the output optical power from the pigtail fiber coupled diode module for the driving current $4 \mathrm{~A}$ that was $3.35 \mathrm{~W}$; i.e. the conversion efficiency was around $83.75 \%$ in the $975 \mathrm{~nm}$ diode laser; while was $85 \%$ in the $808 \mathrm{~nm}$ diode laser; which is considered as high conversion efficiency between the Diode module and the fiber pigtail. From the previous Electrical and Optical characterization measurements; we got the important relation between the output optical power and the input electrical one shown in Figure (6).

Figure (6) shows the relation between the optical output power and the electrical input one in the above curve; while the lower one shows the electrical to optical slope efficiency.

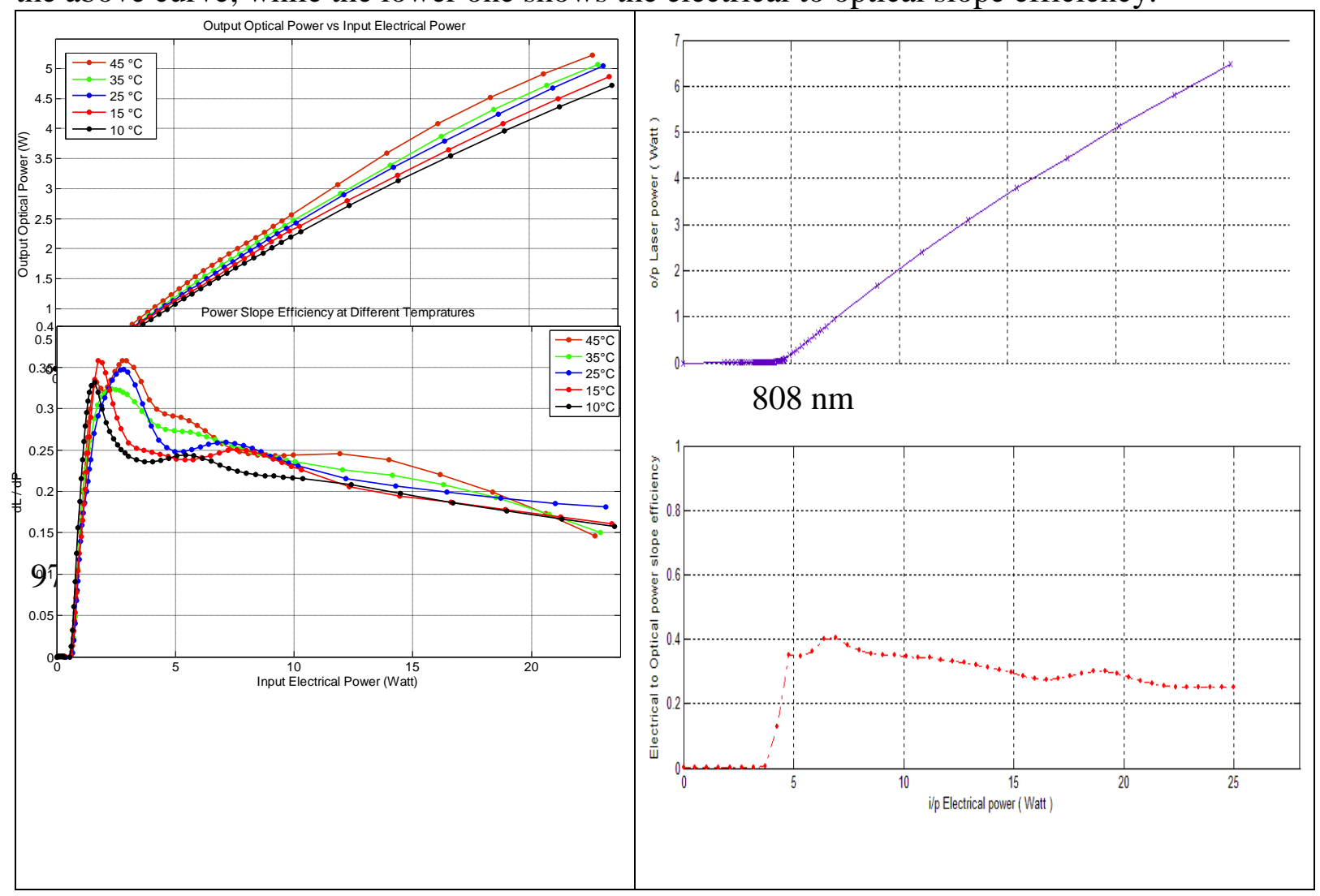

Fig. 6 The high power diode measuring optical characteristics.

From our measurements shown in Figure (6); we have maximum slope efficiency around 36\% for the $975 \mathrm{~nm}$ and $42 \%$ for the $808 \mathrm{~nm}$. The decreasing in the measured efficiency with increasing pumping current shown in Figure (6) occurs because of the ohmic losses in the diode laser increasing with the square of the current [12]; where the output power is in linear relation with the driving current as shown in Figure (5).

\section{The Temperature Effects on the Output Diode Laser Characteristics:}




\subsection{Temperature effect on the lasing threshold point:}

Using the previous experimental setup; with changing the diode operating temperature to different values; and measuring the (L-I) curve in each case, the temperature effect on the output threshold laser can be examined.

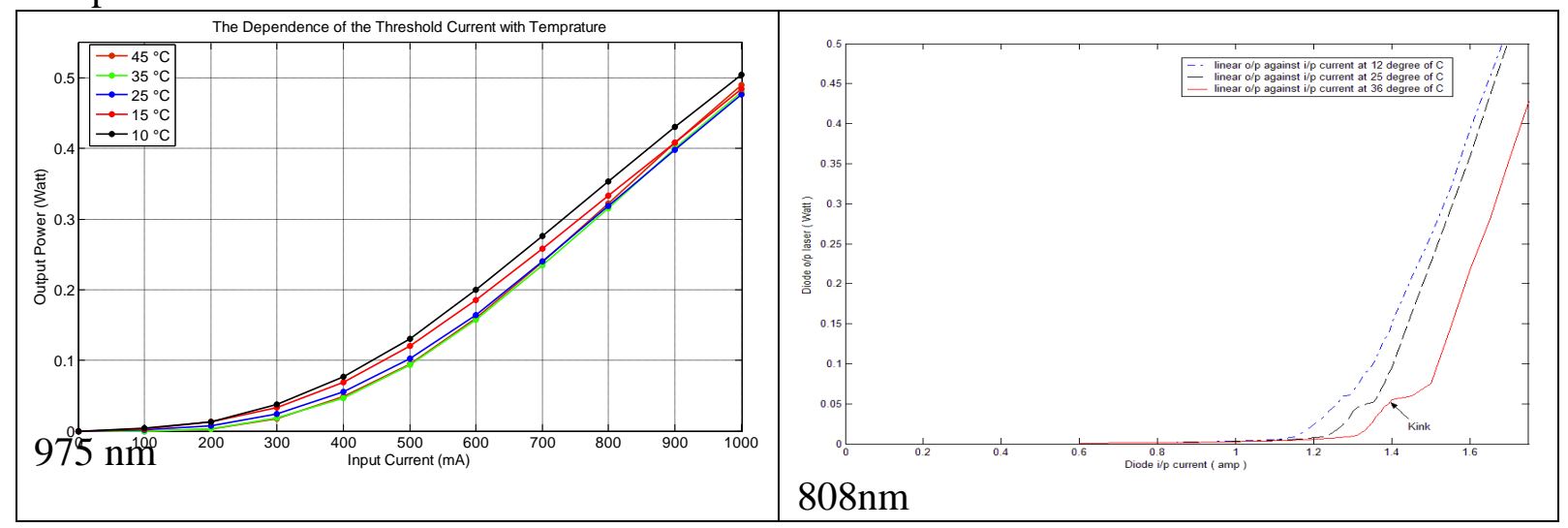

Fig. 7 The laser diode output vs. the driving input current at different temperatures.

Figure (7) shows the laser diode output vs. the driving input current at different values of temperatures, the result illustrates that; the laser threshold output is an increasing function of temperature.

Concerning with the temperature effect on both of threshold lasing and the conversion slope efficiency; and taking into account the measuring results of the electrical characterization shown in Figure (4); we notice that; operating the high power Diode module at the room temperature $\left(25-30^{\circ} \mathrm{C}\right)$ is the best condition. It gives higher slope efficiency with lower consumption electrical power and lower threshold lasing which considered to be an optimum condition producing a maximum output performance.

\subsection{Temperature effects on the wavelength measurements:}

Temperature variation is an important factor that must be monitor if the wavelength will change with the temperature variation or not. Here, for the $975 \mathrm{~nm}$, it was noticed that the wavelength will not change that is a measure of the stability of the diode laser regardless of the temperature variation, while for the $808 \mathrm{~nm}$, it was noticed that the wavelength has a slight shift while changing the temperature.

i) Experimental Setup:

The experimental setup shown in Figure (8) consists of; high resolution Spectrometer, fiber optic integrating spheres, collimated optics, optical density lens, high power pigtail diode module and "Spectra Suite" computer software.

The high resolution Spectrometer (HR4000CG) with a 3648-element linear-array CCD detector that provides better optical resolution produced by "Ocean Optics Inc." shown in Figure (8); providing 200:1100 $\mathrm{nm}$ wavelengths ranging with $0.75 \mathrm{~nm}$ optical resolution (FWHM) is used in our experimental work. "Spectra Suite" spectrometer software is used in analyzing and viewing our measurements.

ii) Experimental Results:

Firstly; we investigate the temperature effect on the laser output wavelength; we measure the diode wavelength at different operating temperatures controlled by the laser diode driver and temperature controller (within the diode operating temperature acceptance $0: 60^{\circ} \mathrm{C}$ ) with a constant driving current $4000 \mathrm{~mA}$. 


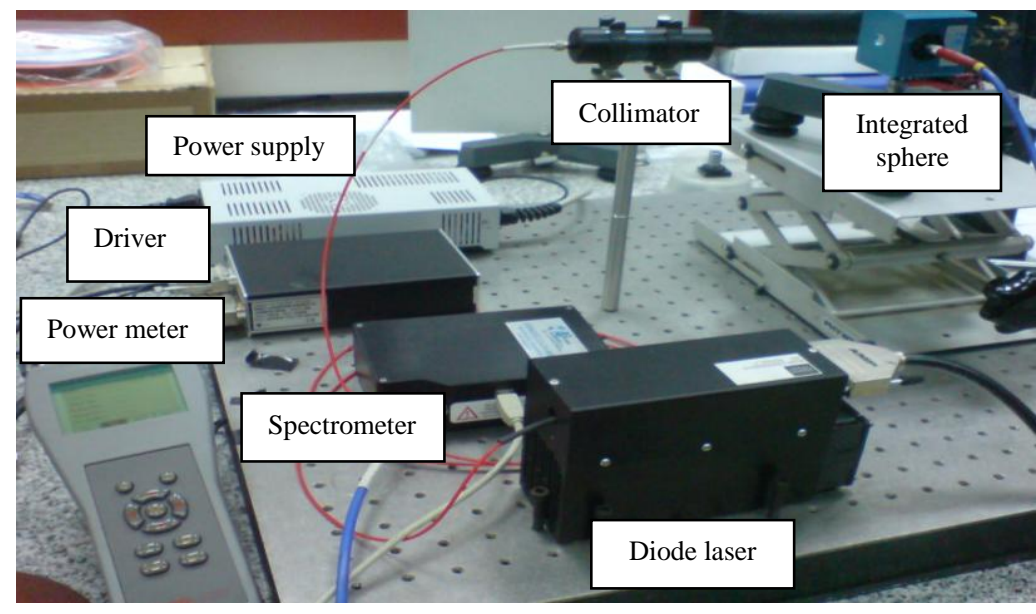

Fig. 8 The experimental setup of the wavelength measurement using high resolution CCD spectrometer ranging from 200:1100 nm.

Figure (9) shows the wavelength of the laser output for the $975 \mathrm{~nm}$ at different temperatures (from 25 up to $35{ }^{\circ} \mathrm{C}$ with step $1{ }^{\circ} \mathrm{C}$ ) at constant driving current of $4 \mathrm{~A}$.

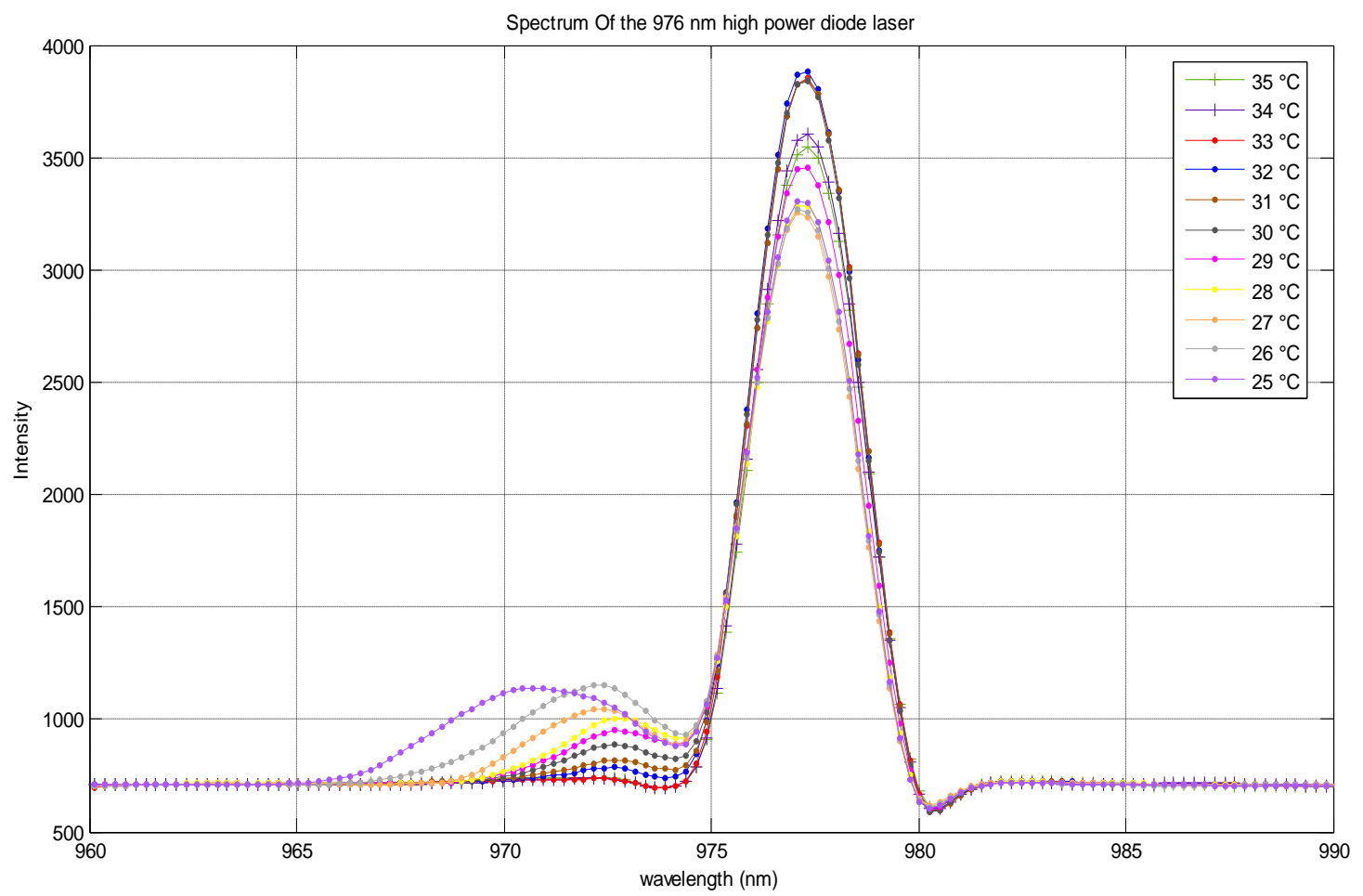

Fig. 9 The wavelength variation according to temperature changed under constant driving current of $4 \mathrm{~A}$.

It was noticed from measurements of Figures $(9,10)$ that the emitted laser wavelength is not affected by the temperature increasing or by the current increment; we did that to investigate the stability of the unit used. From Figure (9) we measured the spectrum of the high power diode laser for the operating current of 4A with different temperatures (from $25{ }^{\circ} \mathrm{C}$ to $35{ }^{\circ} \mathrm{C}$ with step temperature of $1{ }^{\circ} \mathrm{C}$ ) we notice no shift in the output wavelength at $977.34 \mathrm{~nm}$.

Secondly; we measure the wavelength of the diode output at different input driving currents. Figure (10) shows the output wavelength measured at different driving current (from 1A to 7A with step current of $0.5 \mathrm{~A}$ ) for a constant temperature. We repeated this measurement 3 times for 3 different temperatures $\left(25^{\circ} \mathrm{C}, 30^{\circ} \mathrm{C}, 35^{\circ} \mathrm{C}\right)$. 
From our measurements; one can observe that the peak wavelengths for the diode laser are nearly constant for different temperatures at the same driving current); with no shift in the central wavelength.

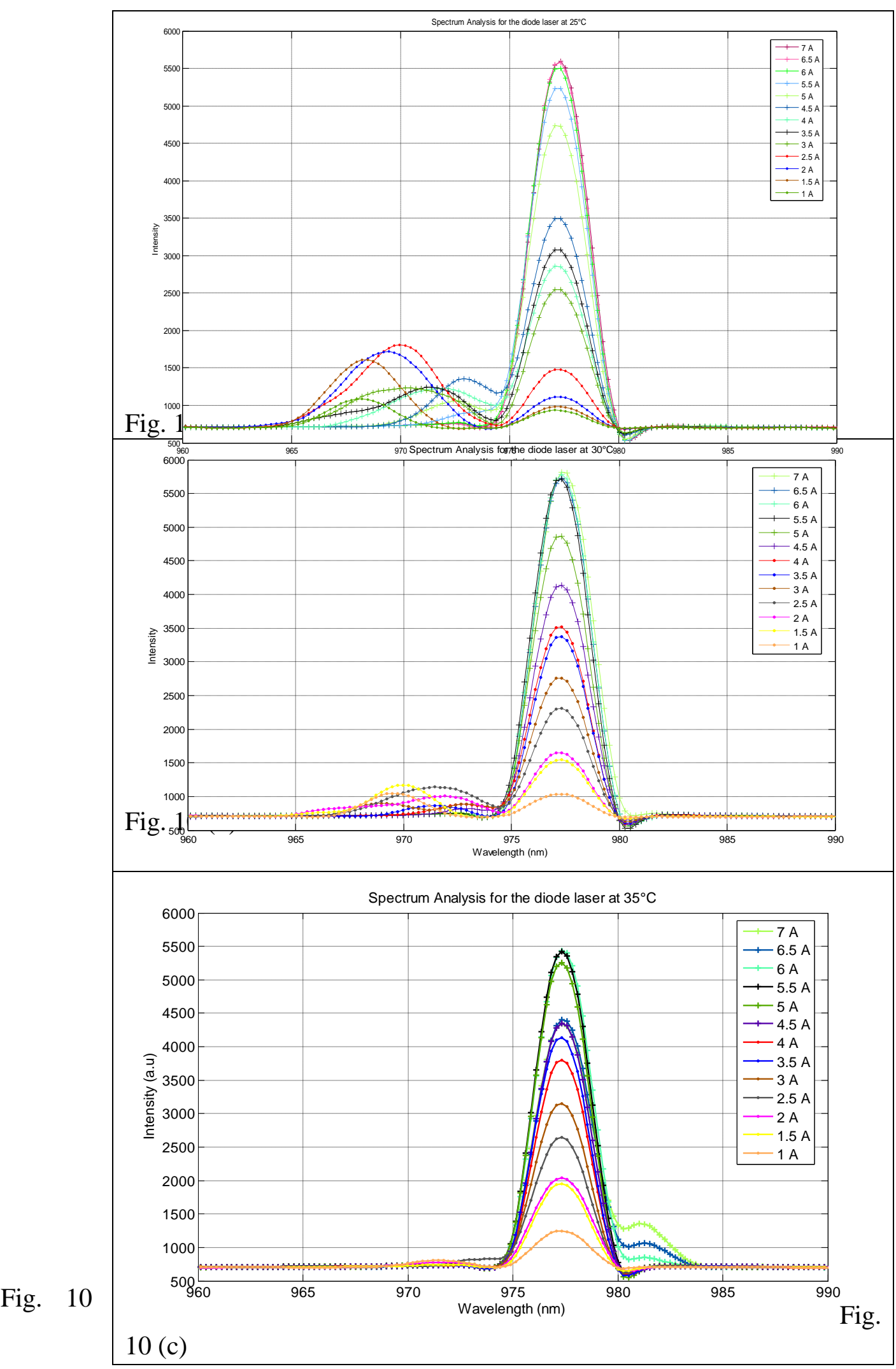


spectrum of the $976 \mathrm{~nm}$ high power diode laser operating at current range from 1A to 7A with step current of $0.5 \mathrm{~A}$ for different temperatures (a) $25{ }^{\circ} \mathrm{C}$, (b) $30{ }^{\circ} \mathrm{C}$, (c) $35^{\circ} \mathrm{C}$.

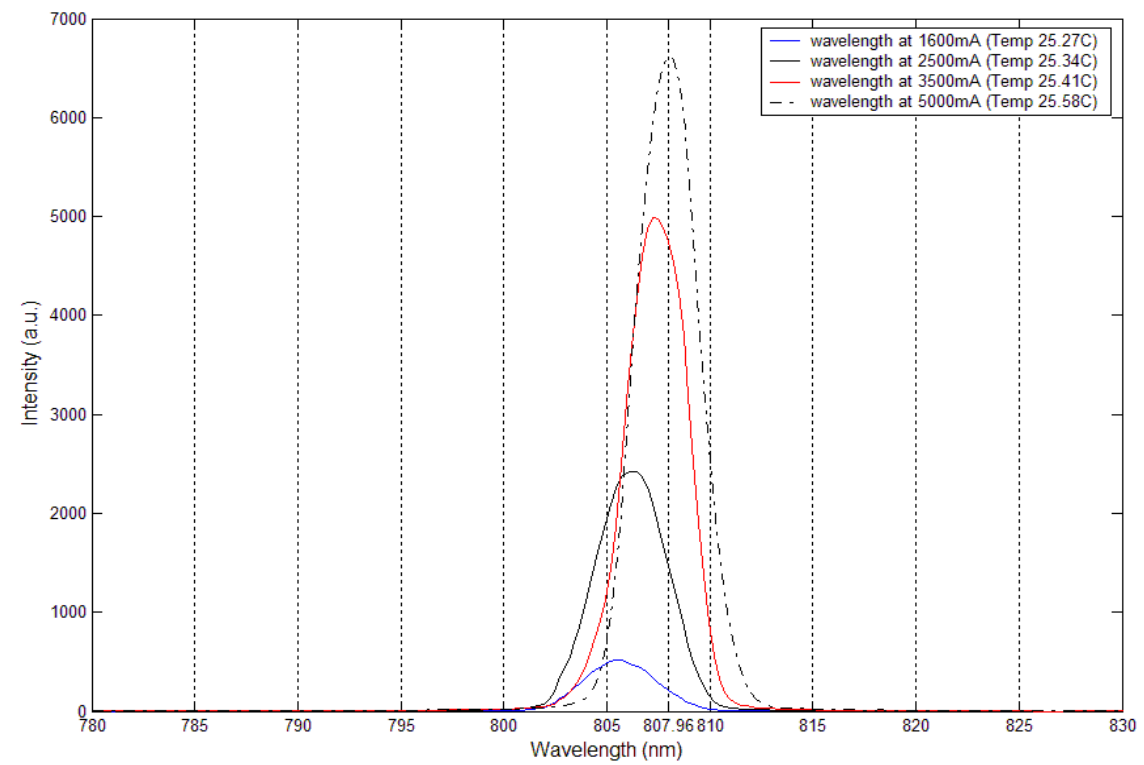

Fig. 11 The spectrum of $808 \mathrm{~nm}$ diode laser according to temperature change with constant driving current $(3.5 \mathrm{~A})$

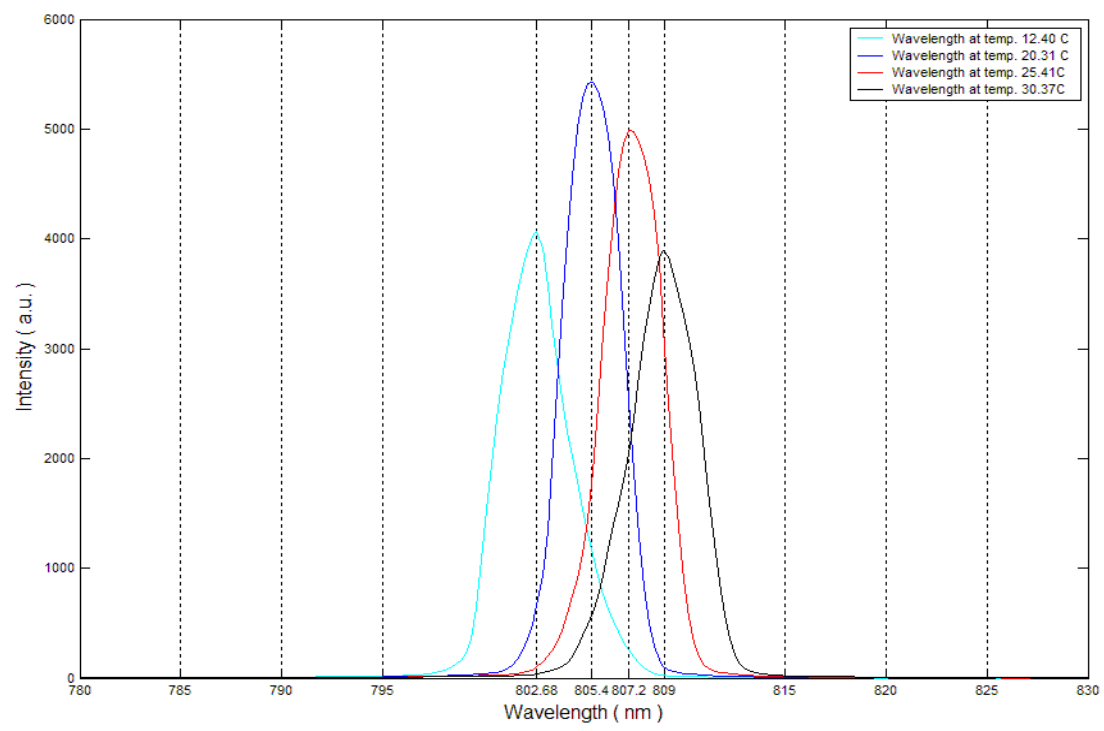

Fig. 12 The spectrum of $808 \mathrm{~nm}$ diode laser; with its peak insignificant shift according to increasing $\mathrm{i} / \mathrm{p}$ currents at constant temperature $\left(25^{\circ} \mathrm{C}\right)$.

Figure (11) shows the wavelength of the laser output at different temperatures $(12,20,25$ and $30^{\circ} \mathrm{C}$ ) at constant driving current $(3.5 \mathrm{~A})$. It was noticed that the emitted laser wavelength is affected by the temperature increasing; and for a good approximation; Diode wavelength will increase by $0.35 \mathrm{~nm} / \mathrm{C}$; which agrees with the published results shown in [25]. This will lead to a suitable technique for controlling the output diode laser wavelength to be typically coincident with the absorbed band for $\mathrm{Nd}^{3+}$ doping materials [26].

From Figure (11) the maximum diode output power obtained at the room temperature $\left(20: 25^{\circ} \mathrm{C}\right)$; which proves that; the ideal temperature for the diode operation is the room temperature. Furthermore; the best useful wavelength band for pumping $\mathrm{Nd}^{3+}$ doped materials was given at the same temperature ranging $\left(20: 25^{\circ} \mathrm{C}\right)$. 
Secondly; we measure the wavelength of the diode o/p at different $\mathrm{i} / \mathrm{p}$ driving currents; nearly at constant temperature. Figure (12) shows the output wavelength measured at different driving current $(1600,2500,3500$ and $5000 \mathrm{~mA})$ for a constant temperature $\left(25^{\circ} \mathrm{C}\right)$. From our measurements; one can observe that the peak wavelengths for the diode laser are nearly constant for different i/p driving currents $(1600,2500,3500$ and $5000 \mathrm{~mA})$; showing a slightly shift towards higher wavelength according to increasing the driving current causes a little increased in the diode temperature. Typical $808 \mathrm{~nm}$ laser o/p is obtained with different driving $\mathrm{i} / \mathrm{p}$ current at constant temperature of $25^{\circ} \mathrm{C}$ with line width of $3.59 \mathrm{~nm}$ measured at FWHM.

The final step in the measurement is the thermal image of the $975 \mathrm{~nm}$ laser beam, which is in the shape of a Gaussian beam and the figure below shows the beam profile of that beam. This measurement was done using the FLUKE Ti20 TERMAL IMAGER as in Figure (13).

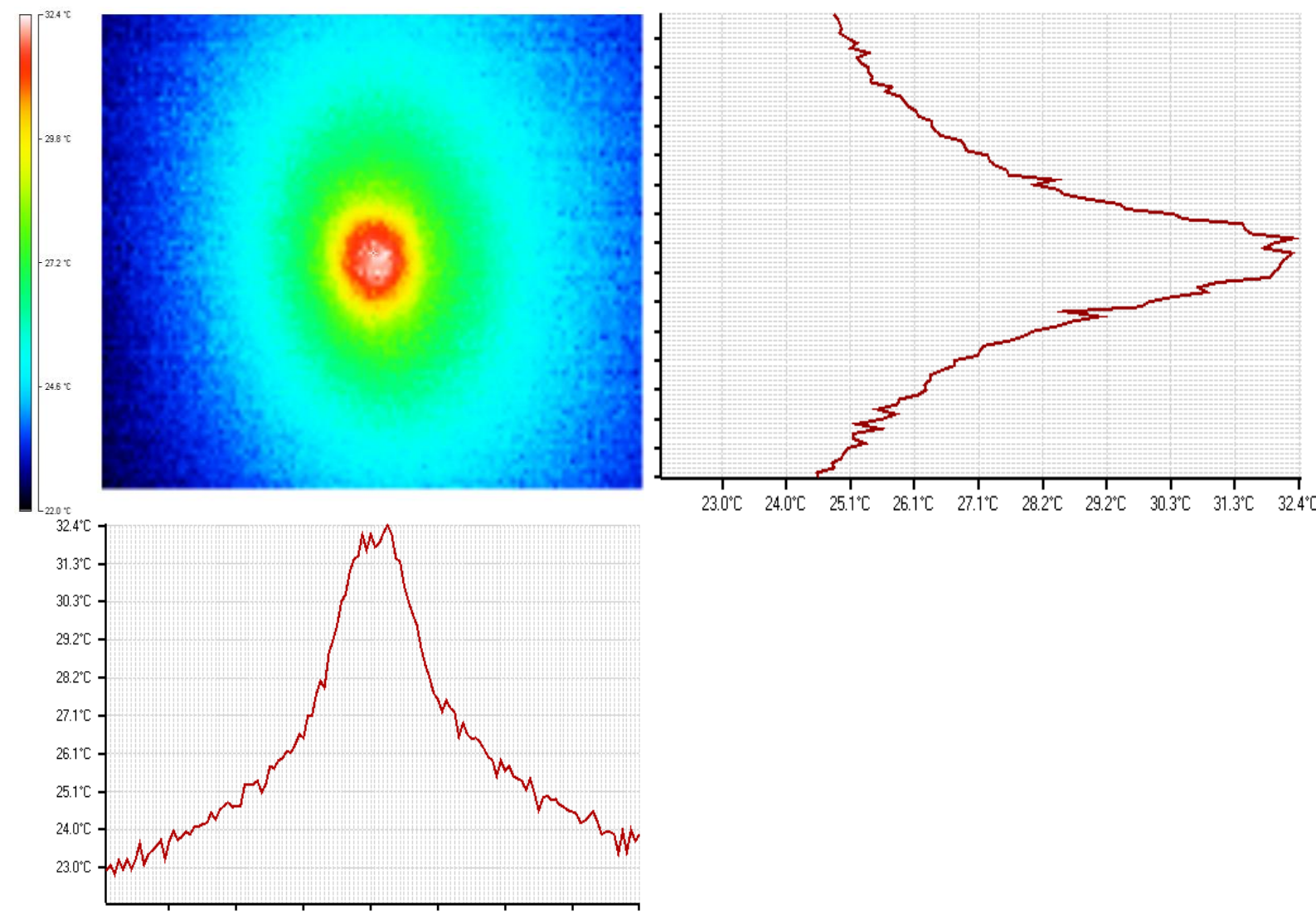

Fig. 13 Output beam profile of diode laser module with stabilized wavelength at $975 \mathrm{~nm}$

\section{Conclusion and Discussions:}

In this paper; the temperature effect on both of threshold lasing and the conversion slope efficiency was measured. Based on the Electrical and Optical characterization measuring results; operation of the high power diode module at the room temperature $\left(25^{\circ} \mathrm{C}\right)$ gave best slope efficiency with low consumption electrical power and low in lasing threshold; which is considered as an optimum condition that gives the maximum output performance.

Maximum slope efficiency around $35.84 \%$ for the $975 \mathrm{~nm}$ with a maximum electrical to optical conversion efficiency of $25 \%$ while it was $42 \%$ for the $808 \mathrm{~nm}$ with a maximum electrical to optical conversion efficiency of $28 \%$ was measured. The emitted laser wavelength was not affected by the temperature increasing. 
Moreover; the appropriate wavelength that useful for pumping $\mathrm{Yb}^{3+}$ doped fiber (around $975 \mathrm{~nm})$ was obtained at the temperature range of $\left(25^{\circ} \mathrm{C}\right)$. The peak wavelengths for the diode laser were found to be nearly constant for different operating temperatures. Typical $975 \mathrm{~nm}$ diode laser output was obtained with different driving input current at constant temperature of $25^{\circ} \mathrm{C}$ with line width of $1.3 \mathrm{~nm}$ for the $975 \mathrm{~nm}$, and $3.59 \mathrm{~nm}$ for the $808 \mathrm{~nm}$ at FWHM.

For the $808 \mathrm{~nm}$ diode laser module, which was not provided with volume holographic grating (VHG) the emitted laser wavelength was affected by the temperature or driving current increase; and for a good approximation; diode wavelength was increased by $0.35 \mathrm{~nm} /{ }^{\circ} \mathrm{C}$, while for the $975 \mathrm{~nm}$ diode laser module provided with (VHG), the emitted laser was not shifted according to the advantages of volume holographic grating, which enhance the wavelength stability for either change of driving current or wide range of temperatures.

By providing wavelength-selective feedback into a laser diode (LD), a VHG can lock the lasing wavelength to that of grating. This serves to lower the temperature dependence of the wavelength, narrow the spectrum, reduce the aging-related wavelength changes, and in the case of diode arrays, lock each emitter to the same wavelength, producing a much narrower combined spectrum than that in the unlocked arrays [27].

VHGs can have narrow spectral response (below $0.1 \mathrm{~nm}$ ) and a small spatial acceptance angle (below $0.1^{\circ}$ ). A small VHG formed by the interference of two collimated beams acts as a spectral filter with diffraction efficiency approaching 100\%, narrow bandwidth, and precise central frequency. These features are important for several spectral filter applications such as laser line rejection, separating and combining beams of different wavelengths for spectral analysis, signal detection, power combining, wavelength-selection, routing and switching, and improving LD emission characteristics by locking and stabilizing the wavelength [27].

\section{References:}

[1] R. Paschotta , "www.rp.photonics.com", (c) 2007.

[2] Ashraf F. El-Sherif, "Optimization of a high power fibre coupled diode end pumped Nd:YVO4 solid state laser", in "Solid State Lasers XVIII", LASE 2009, Photonic West Conference, Poc. of SPIE Vol. 7193, California, USA, Jan. 2009

[3] A. J. Antończak, J. Z. Sotor, M. Matysiak and K. M. Abramski, " Blue 473-nm solid state diode pumped Nd:YAG/BiBO microchip laser", J. of Opto-Electronic Review, Vol. 18, No. 1, 71-74, 2009.

[4] XU Yi-Ting, XU Jia-Lin, CUI Qian-Jin, XIE Shi-Yong, LU Yuan-Fu, PENG Qin-Jun, CUI Da-Fu, and XU Zu-Yan "High Efficiency Multi-kW Diode-Side-Pumped Nd:YAG Laser with Reduced Thermal Effect", CHIN. PHYS. LETT., Vol. 27, No. 2, 2010.

[5] Yunsong Joeng, Youngbok Kim, Andreas Liem, Klaus Moerl, Sven Hoefer, Andreas Tuennermann, and Kyunghwan Oh., " Q-switching of Yb3+-doped fiber laser using a novel micro-optical waveguide on microactuating platform light modulator", 12, Vol. 13, No. 25, Optics Express, 10302, December 2005.

[6] R Duchowicz1,2, N A Russo1, E Sicre3 and M V Andr'es, "Q-switching of an erbiumdoped fibre laser modulated by a Bragg grating fixed to a piezoelectric", J. Opt. A: Pure Appl. Opt., 5, S216-S220, 2003.

[7] David Sabourdy, David Bouyge, Aurelian Crunteanu, Pierre Blondy, Vincent Couderc, Jérôme Lhermite, Vincent Kermène, Agnès Desfarges-Berthelemot, Ludovic Grossard, and Alain Barthélemy, "Novel active Q-switched fiber laser based on electrostatically actuated micro-mirror system", Vol. 14, No. 9, Optics Express, May 2006.

[8] Matthew Leigh, Wei Shi, Jie Zong, Jiafu Wang, and Shibin Jiang, "Compact, singlefrequency all-fiber Q-switched laser at 1 $\mu \mathrm{m} "$, Vol. 32, No. 8, Optics Letters, April 15, 2007.

[9] YOSHIDA Hidetsugu, TSUBAKIMOTO Koji, FUJITA Hisanori, NAKATSUKA Masahiro, MIYANAGA Noriaki, and IZAWA Yasukazu, "Front-end system used in PM-Yb fiber laser for high-peak power laser system", J. of Physics: Conference Series 112, 2008. 
[10] S. W. Harun, M. C. Paul, M. R. A. Moghaddam, S. Das, R. Sen, A. Dhar, M. Pal, S. K. Bhadra and H. Ahmad, "Diode-pumped $1028 \mathrm{~nm}$ Ytterbium-doped fiber laser with near 90\% slope efficiency", J. of Laser Physics, VOL 20, No. 3, 656-660, 2009.

[11] S. I. Kablukov, E. I. Dontsova, V. A. Akulov, A. A. Vlasov and S. A. Babin, "Frequency doubling of Yb-doped fiber laser to 515 nm", J. of Laser Physics, VOL 20, No. 2, 360-364, 2010 .

[12] W.STREIFER, D.R.SCIFRES, G.L.HARNAGEL, D.F.WELCH, J.BERGER and M.

SAKAMOTO, "Advances in Diode Laser pumps" IEEE, Q.E vol24.1988.

[13] O. Svelto, "PRINCIPLES OF LASERS", Springer, fourth edition, page 201-224, 2009.

[14] W. Koechner, "Solid State Laser Engineering", Springer-Berlin, fifth edition, Page 4763,134-147,463-469. 1999.

[15] Bernd Koller, Thomas Brand, Matthias Haag, and Jens Biesenbach, "Wavelength stabilized high power diode laser modules", DILAS Diodenlaser GmbH, 2009.

[16] Maiwald, M. Erbert, G. Klehr, A. Sumpf, B. Wenzel, H. Laurent, T. Wiedmann, J. Kronfeldt, H.D. Schmidt, "Reliable operation of $785 \mathrm{~nm}$ DFB diode lasers for rapid Raman spectroscopy", High-power diode laser technology and applications V, Proc. of the SPIE, VOL 6456, pages 64560W, 2007.

[17] M. Kanskar; J. Cai; C. Galstad; Y. He; S. H. Macomber; E. Stiers; S. R. TatavartiBharatam; D. Botez; L. J. Mawst, " High power conversion efficiency and wavelengthstabilized narrow bandwidth 975nm diode laser pumps", Laser Source and System Technology for Defense and Security II, Proc. of the SPIE, Vol. 6216, 2006.

[18] LAURENT VAISSIÉ, TOM STEELE, PAUL T. RUDY, "High-power diode lasers advance pumping applications", Laser Focus World, PennWell Corporation, June 2008.

[19] Friedrich bachmann, Peter loosen and Reinhart Poprawe, " High Power Diode Lasers: Technology and Applications", Springer series in optical sciences, pp. 121-123, pp. 162-174, 2007.

[20] Haag, Matthias; Köhler, Bernd; Biesenbach, Jens; Brand, Thomas, "Novel highbrightness fiber coupled diode laser device", High-Power Diode Laser Technology and Applications V, Proc. of the SPIE, Volume 6456, pp. 64560T, 2007.

[21] B. L. Volodin, S. V. Dolgy, E. D. Melnik, E. Downs, J. Shaw, and V. S. Ban, "Wavelength stabilization and spectrum narrowing of high-power multimode laser diodes and arrays by use of volume Bragg gratings", Optics Letters, Vol. 29, Issue 16, pp. 1891-1893, 2004.

[22] Christophe Moser and Gregory Steckman, Ondax Inc., " Filters to Bragg About Volume holographic gratings offer distinctive filter qualities", Photic Spectra, pp. 82, June 2005.

[23] G.B. Venus, A. Sevian, V.I. Smirnov, L.B. Glebov. "High-brightness narrow-line laser diode source with volume Bragg-grating feedback", High-Power Diode Laser Technology and Applications III, Proc. of SPIE, VOL. 5711, 166-176, 2005.

[24] C. Schnitzler, S. Hambuecker, O. Ruebenach, V. Sinhoff, G. Steckman, L. West, C. Wessling, D. Hoffmann, " Wavelength stabilization of HPDL array: fast-axis collimation optic with integrated VHG', High-Power Diode Laser Technology and Applications V, Proc. of SPIE 6456, 645612, 2007.

[25] C. A. Wang and S. H .Groves "New materials for Diode pumping solid state Lasers" IEEE, Q.E.vol28.1992.

[26] Juergen Pfaff, "Method of controlling diode laser wavelength in a DPSSL", Patent No.US6, 292, 498B1, 2001

[27] Gregory J. Steckman, Wenhai Liu Platz, R. Schroeder, D. Moser, C. Havermeyer, F., "Volume Holographic Grating Wavelength Stabilized Laser Diodes", IEEE Journal of Selected Topics in Quantum Electronics, VOL. 13, NO. 3, pp. 672-678, 2007. 